\title{
Feedback linearizing control of a magnetically coupled multiport dc-dc converter for automotive applications
}

\author{
Josep M. Olm, Enric Fossas, Victor Repecho, Arnau Dòria-Cerezo and Robert Griñó \\ Institute of Industrial and Control Engineering \\ Universitat Politècnica de Catalunya \\ Barcelona, Spain \\ \{josep.olm,enric.fossas,victor.repecho.del,arnau.doria,roberto.grino $\} @$ upc.edu
}

\begin{abstract}
This paper presents a feedback linearization-based control strategy for a a magnetically coupled multiport dc-dc converter aimed at automotive applications. Essentially, a transformation of control and state variables is proposed in such a way that the resulting system becomes linear and decoupled. Then, a conventional PI controller is devised that renders asymptotic stability, as well as robustness to resistive load uncertainty and presence of constant power loads. The inversion of the control transformation, which involves nonlinear terms, is carried out using linear approximations with no significant performance decay. Hence, simplicity and efficiency are the main features of the control design. The proposal is validated via realistic numerical simulations.

Index Terms-Multiport dc-dc converter, feedback linearization, decoupling, PI control, automotive applications
\end{abstract}

\section{INTRODUCTION}

In the last years two main challenges have raised up in the automotive sector: on the one hand we have $\mathrm{CO}_{2}$ reduction, which implies a weight reduction and an efficiency increase of the vehicle components [1]; on the other hand we have the customer's requirements, mainly in terms of performance and security. Consequently, the use of electrified systems has emerged as an alternative to be taken into account by OEMs (Original Equipment Manufacturers). This new tendency does not only consider the electrification of the power train (i.e. electrical and hybrid vehicles), but it is also evident in many auxiliary devices that are increasingly being electrified [2].

However, a major electrification of vehicles entails issues related to on-board dc micro-grids. This implies the necessity of designing: (i) controllers ensuring grid stability in the face of the connection of constant power loads (CPLs), as well as good robustness and efficiency properties, and (ii) optimization algorithms for the dc micro grid.

This paper considers a dc multi-bus with the most used voltages in automotive applications, namely, $400 \mathrm{~V}$ for the power train [3], [4], and $48 \mathrm{~V}$ and $12 \mathrm{~V}$, for auxiliary devices, composed by only one storage element (batteries), see Figure 1. The main advantage of using only one battery and one power converter is size and weight reduction.

The dc buses are connected by a magnetically coupled multiport dc-dc converter (400/48/12V dc). This kind of dc-dc

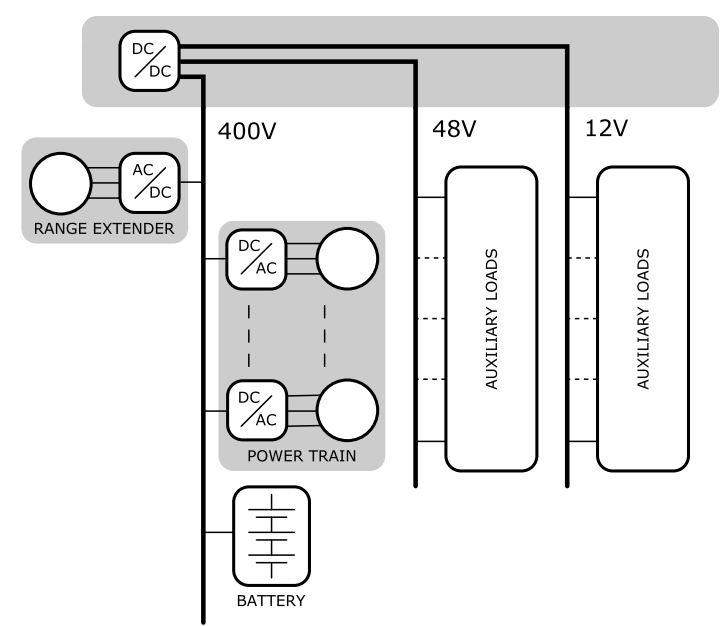

Fig. 1. Scheme of a dc-dc multi-port converter.

converters have recently attracted the interest of researchers for automotive applications [5], but also for connecting different dc networks. Other examples include electric aircrafts [6], satellites [7], energy management systems [8], dc grids [9]...

Usually, the control of magnetically coupled dc-dc converters is restricted to classical PI controllers, see examples in [6], [10], and PI controllers based on the Generalized State-Space Averaging (GSSA) decomposition [11]. Only few references propose alternatives such as decoupling methods [12], [13] or compensators, a two-pole one-zero compensator in [14] or three-pole two-zero compensator in [15]. In general, the proposed control strategies are based on a linear approximated model. The aim of this paper is to develop a feedback linearization-based control strategy for a dc multi-bus and a multiport power converter that provides energy management among the dc buses, and ac/dc charging functionalities. Its main advantage with respect to linear techniques is that the control design includes the inherent nonlinearities of the model. Indeed, an appropriate control and state variables transformation is proposed that linearizes and decouples the system, and a PI controller rendering asymptotic stability and 
robustness to resistive load changes and presence of CPLs is devised. Then, the control transformation, which involves nonlinear terms, is inverted using linear approximations, and no major performance decay is observed. The obtained control algorithm is validated via realistic numerical simulations.

The remainder of the paper is organized as follows. The mathematical model of the multiport dc-dc converter is presented in Section II. The feedback linearizing control strategy is proposed in Section III, and issues related to the inversion of the control law are discussed in Section IV. Simulation results are gathered in Section V and, finally, conclusions and some suggestions for further research are drawn in Section VI.

\section{Mathematical Model}

Figure 2 shows the schematic of the magnetically coupled multiport dc-dc converter. It consists of a high frequency transformer with $n$ ports together with a full-bridge converter and a capacitor in its dc side, where $v_{k}$ are the dc voltages, $i_{p k}$ refer to the dc currents, $C_{k}$ are the capacitors, $i_{k}, v_{k}^{\prime}$ are the high frequency currents and voltages in the transformer, respectively, $N_{k}$ are the number of coils in each winding, and subindex $k \in\{1,2, \ldots, n\}$ refers to the port number. From the behavioural approximation [16] the dc voltage dynamics can be modelled as

$$
C_{k} \dot{v}_{k}=i_{p k}-\frac{1}{\omega_{1}} \sum_{l=1, l \neq k}^{n} \frac{v_{l}}{\alpha_{k l} L_{k l}^{\prime}} \delta_{k l}\left(1-\frac{\left|\delta_{k l}\right|}{\pi}\right),
$$

where $\omega_{1}=\frac{2 \pi}{T}$ is the fundamental frequency of the periodic voltages and currents in the transformer, $\alpha_{k l}$ is the transformer turn ratio of the $k$-th port with respect to the $l$-th port, $L_{k l}^{\prime}$ is the meshed-transformed linking inductance between port $k$ and any $l$-th port, and $\delta_{k l}=\theta_{l}-\theta_{k}$, where the $\theta$ 's are the phase shifts of the modulated voltages.

Let us then consider a three dc bus system: a high voltage dc bus (nominal voltage $400 \mathrm{~V}$ ) and two low voltage buses (nominal voltages $48 \mathrm{~V}$ and $12 \mathrm{~V}$ ). The voltage at the $400 \mathrm{~V}$ bus is assumed constant (mainly, due to the usual high capacity battery in these buses) and the two other buses have several loads connected to them. Setting the phase shift for port 1 as the reference one, i.e., $\theta_{1}=0$, the system dynamics is given by:

$$
\begin{aligned}
C_{2} \dot{v}_{2}= & -\frac{v_{2}}{R_{2}}-\frac{P_{2}}{v_{2}}+\frac{E_{1}}{\omega_{1} \alpha_{12} L_{12}^{\prime}} \theta_{2}\left(1-\frac{\left|\theta_{2}\right|}{\pi}\right)+ \\
& -v_{3} \frac{1}{\omega_{1} \alpha_{23} L_{23}^{\prime}}\left(\theta_{3}-\theta_{2}\right)\left(1-\frac{\left|\theta_{3}-\theta_{2}\right|}{\pi}\right) \\
C_{3} \dot{v}_{3}= & -\frac{v_{3}}{R_{3}}-\frac{P_{3}}{v_{3}}+\frac{E_{1}}{\omega_{1} \alpha_{13} L_{13}^{\prime}} \theta_{3}\left(1-\frac{\left|\theta_{3}\right|}{\pi}\right)+ \\
& +v_{2} \frac{1}{\omega_{1} \alpha_{32} L_{32}^{\prime}}\left(\theta_{3}-\theta_{2}\right)\left(1-\frac{\left|\theta_{3}-\theta_{2}\right|}{\pi}\right),
\end{aligned}
$$

where $v_{2}$ is the voltage of the $48 \mathrm{~V}$ bus, $v_{3}$ is the voltage of the $12 \mathrm{~V}$ bus, and the phase shifts in ports 2 and 3 , namely $\theta_{2}, \theta_{3}$, respectively, are the control variables, with $\theta_{i} \in\left[-\frac{\pi}{2}, \frac{\pi}{2}\right]$, $i \in\{2,3\}$.

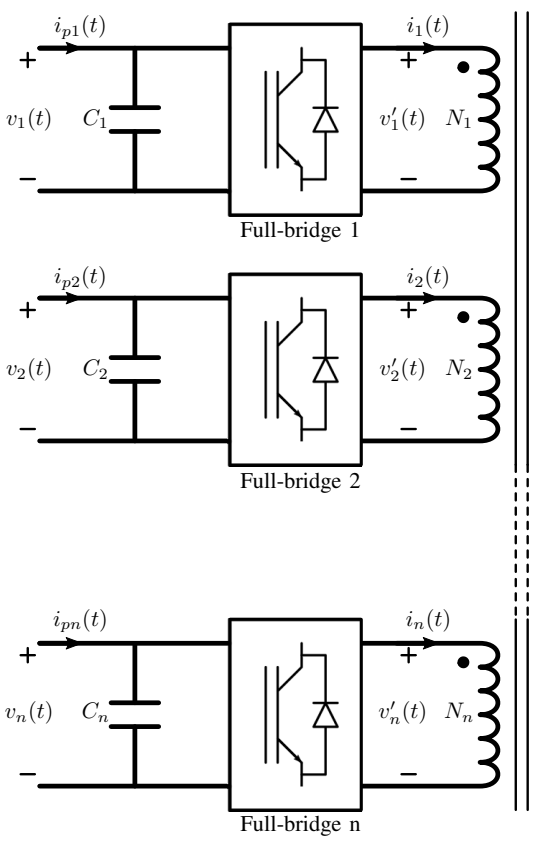

Fig. 2. Multiport dc-dc converter.

The control objectives are to regulate the voltages $v_{2}$ and $v_{3}$ to the reference values, $v_{2}^{*}=48 \mathrm{~V}$ and $v_{3}^{*}=12 \mathrm{~V}$, in spite of the load variations in the buses (described by $R_{i}, P_{i}$, respectively). For control design purposes it is assumed that the voltages at the buses are available for measure.

Let

$$
\begin{aligned}
f_{i}\left(\theta_{i}\right) & =\frac{E_{1}}{\omega_{1} \alpha_{1 i} L_{1 i}^{\prime}} \theta_{i}\left(1-\frac{\left|\theta_{i}\right|}{\pi}\right), \quad i=2,3 \\
\lambda_{i} & =\frac{1}{\omega_{1} \alpha_{i j} L_{i j}^{\prime}}, \quad i=2,3, \quad i \neq j, \\
g\left(\theta_{2}, \theta_{3}\right) & =\left(\theta_{3}-\theta_{2}\right)\left(1-\frac{\left|\theta_{3}-\theta_{2}\right|}{\pi}\right) .
\end{aligned}
$$

Then, (2) becomes:

$$
\begin{aligned}
& C_{2} \dot{v}_{2}=-\frac{v_{2}}{R_{2}}-\frac{P_{2}}{v_{2}}+f_{2}\left(\theta_{2}\right)-\lambda_{2} v_{3} g\left(\theta_{2}, \theta_{3}\right) \\
& C_{3} \dot{v}_{3}=-\frac{v_{3}}{R_{3}}-\frac{P_{3}}{v_{3}}+f_{3}\left(\theta_{3}\right)+\lambda_{3} v_{2} g\left(\theta_{2}, \theta_{3}\right) .
\end{aligned}
$$

\section{Control StRATEGy}

The selected control law uses a feedback linearization approach. Let us consider the change of state variables

$$
\xi_{i}=v_{i}^{2}, \quad i=2,3,
$$

and the change of control variables

$$
\begin{aligned}
& u_{2}=v_{2} f_{2}\left(\theta_{2}\right)-\lambda_{2} v_{2} v_{3} g\left(\theta_{2}, \theta_{3}\right) \\
& u_{3}=v_{3} f_{3}\left(\theta_{3}\right)+\lambda_{3} v_{2} v_{3} g\left(\theta_{2}, \theta_{3}\right) .
\end{aligned}
$$

Then, (3) boils down to the linear, decoupled system

$$
\dot{\xi}_{i}=-\frac{2}{R_{i} C_{i}} \xi_{i}-\frac{2 P_{i}}{C_{i}}+\frac{2}{C_{i}} u_{i}, \quad i=2,3 .
$$



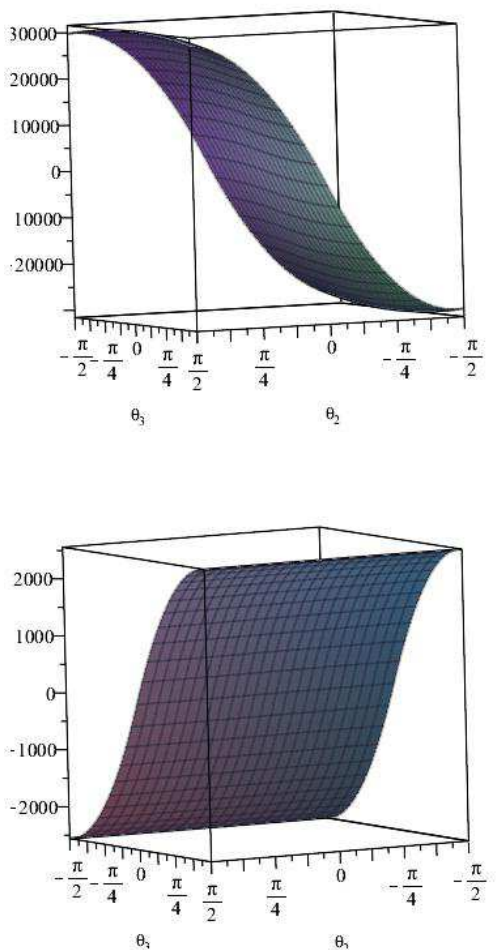

Fig. 3. Top: $u_{2}=u_{2}\left(\theta_{2}, \theta_{3}\right)$; bottom: $u_{3}=u_{3}\left(\theta_{2}, \theta_{3}\right)$.

Let $\xi_{i}^{*}$ denote the reference for the state variable $\xi_{i}, i=2,3$. Then, consider the following PI controller:

$$
\begin{aligned}
u_{i} & =-k_{p i} \xi_{i}+k_{z i} z_{i} \\
\dot{z}_{i} & =\xi_{i}^{*}-\xi_{i},
\end{aligned}
$$

with $i=2,3$. The closed-loop system is therefore given by

$$
\begin{aligned}
& \dot{\xi}_{i}=-\frac{2}{C_{i}}\left(\frac{1}{R_{i}}+k_{p i}\right) \xi_{i}-\frac{2 P_{i}}{C_{i}}+\frac{2 k_{z i}}{C_{i}} z_{i}, \\
& \dot{z}_{i}=\xi_{i}^{*}-\xi_{i},
\end{aligned}
$$

$i=2,3$. This system has a single equilibrium point, namely $\left(\xi_{2}^{*}, z_{2}^{*}, \xi_{3}^{*}, z_{3}^{*}\right)^{\top}$, with

$$
z_{i}^{*}=\frac{1}{k_{z i}}\left[\left(\frac{1}{R_{i}}+k_{p i}\right) \xi_{i}^{*}+P_{i}\right], \quad i=2,3 .
$$

Now, defining $e_{i}=\xi_{i}-\xi_{i}^{*}, e_{z i}=z_{i}-z_{i}^{*}, i=2,3$, (7) becomes linear:

$$
\begin{aligned}
\dot{e}_{i} & =-\frac{2}{C_{i}}\left(\frac{1}{R_{i}}+k_{p i}\right) e_{i}+\frac{2 k_{z i}}{C_{i}} e_{z i} \\
\dot{e}_{z i} & =-e_{i},
\end{aligned}
$$

with $i=2,3$. Finally, it follows straightforward that its characteristic polynomial is

$$
p(\chi)=\prod_{i=2}^{3}\left[\chi^{2}+\frac{2}{C_{i}}\left(\frac{1}{R_{i}}+k_{p i}\right) \chi+\frac{2 k_{z i}}{C_{i}}\right],
$$

which has all its roots in the left complex plane if and only if

$$
\frac{1}{R_{i}}+k_{p i}>0, \quad k_{z i}>0, \quad i=2,3 .
$$
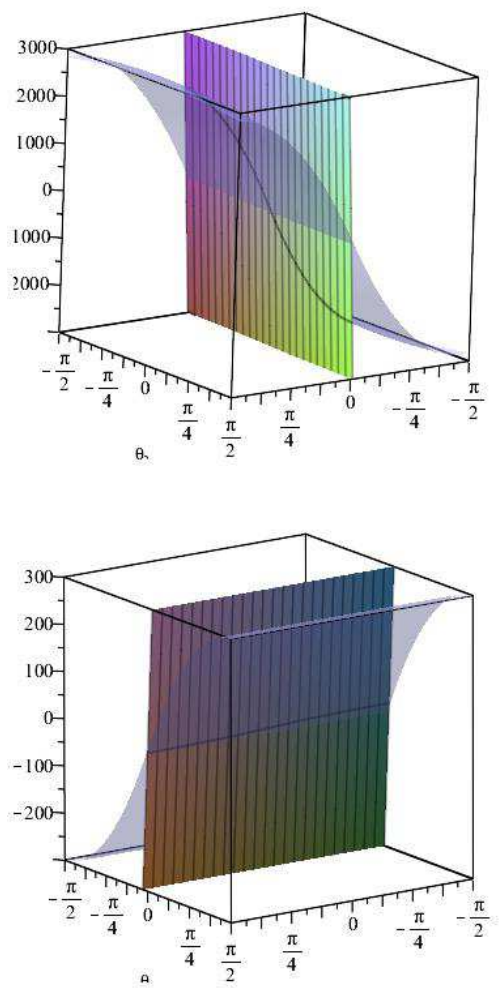

Fig. 4. Zoom of Figure 3. Top: $u_{2}=u_{2}\left(\theta_{2}, \theta_{3}\right)$; bottom: $u_{3}=u_{3}\left(\theta_{2}, \theta_{3}\right)$.

Then, $\left(\xi_{2}^{*}, z_{2}^{*}, \xi_{3}^{*}, z_{3}^{*}\right)^{\top}$ is a globally asymptotically stable equilibrium, this showing that the control law (6) is robust to parametric uncertainty on $R_{i}, P_{i}, i=2,3$.

Remark. The main issue with this control law is in the inversion of the change of variables (4), which is analyzed in next Section.

\section{INVERSION OF THE CONTROL LAW}

The control law (4) is highly nonlinear, as it involves absolute value terms. In order to carry out the inversion, i.e. to obtain $\theta_{i}=\theta_{i}\left(u_{2}, u_{3}\right), i=2,3$, we may first notice from Figure 3 that the $\mathbb{R}^{3}$ surfaces defined in (4) have a large range within its domain, $\theta_{i} \in\left[-\frac{\pi}{2}, \frac{\pi}{2}\right]$. However, taking into account that $u_{i}$ has power units, and that the range of admissible power is, approximately, $u_{2} \in[0,2400 \mathrm{~W}], u_{3} \in[0,600 \mathrm{~W}]$, the zoom in Figure 4 discloses a linear behavior of the surfaces in such region.

This is consistent with the fact that, although

$$
\theta\left(1-\frac{|\theta|}{\pi}\right)
$$

is nicely approximated by $\frac{\pi}{4} \sin \theta$ in $\left[-\frac{\pi}{2}, \frac{\pi}{2}\right]$, the identity, $\theta$, does better in a neighbourhood of $\theta=0$, as plotted in Figure 5. Hence, the inversion will be performed from the linear approximation of the change of variables (4), namely,

$$
\begin{aligned}
& u_{2} \approx v_{2}\left(k_{2} \theta_{2}-\lambda_{2} v_{3}\left(\theta_{3}-\theta_{2}\right)\right) \\
& u_{3} \approx v_{3}\left(k_{3} \theta_{3}+\lambda_{3} v_{2}\left(\theta_{3}-\theta_{2}\right)\right),
\end{aligned}
$$



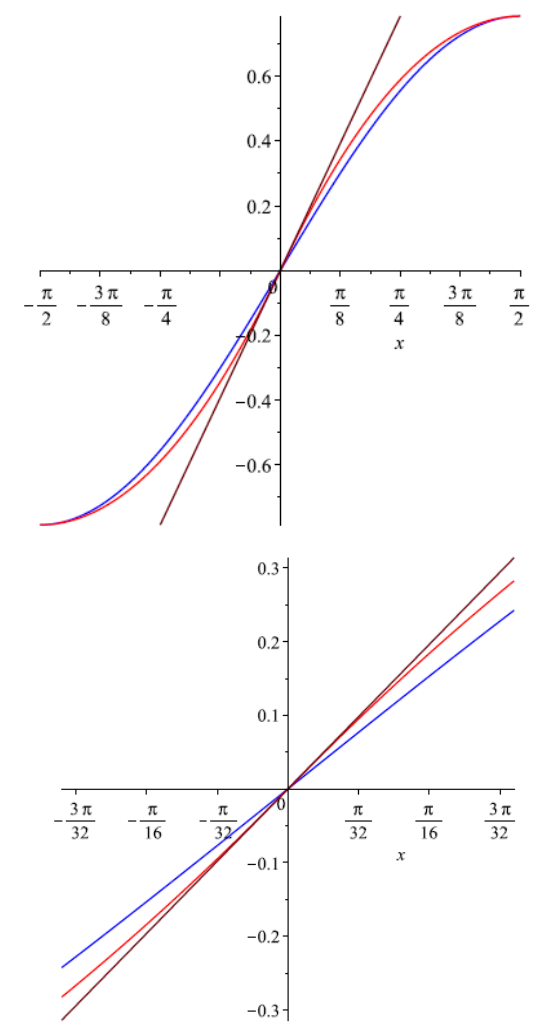

Fig. 5. Red: $\theta\left(1-\frac{|\theta|}{\pi}\right)$; blue: $\frac{\pi}{4} \sin \theta$; brown: $\theta$. Top: $\theta \in\left[-\frac{\pi}{2}, \frac{\pi}{2}\right]$; bottom: $\theta \in\left[-\frac{3 \pi}{32}, \frac{3 \pi}{32}\right]$.

with

$$
k_{i}=\frac{E_{1}}{\omega_{1} \alpha_{1 i} L_{1 i}^{\prime}}, \quad i=2,3
$$

Therefore:

$$
\begin{gathered}
\theta_{2} \approx \frac{\left(\lambda_{3}+\frac{k_{3}}{v_{2}}\right) u_{2}+\lambda_{2} u_{3}}{\lambda_{3} k_{2} v_{2}+\lambda_{2} k_{3} v_{3}+k_{2} k_{3}} \\
\theta_{3} \approx \frac{\lambda_{3} u_{2}+\left(\lambda_{2}+\frac{k_{2}}{v_{3}}\right) u_{3}}{\lambda_{3} k_{2} v_{2}+\lambda_{2} k_{3} v_{3}+k_{2} k_{3}},
\end{gathered}
$$

where the control actions $u_{i}$ are defined in (6).

\section{Simulation Results}

The numerical results have been obtained using MatlabSimulink, where the power converter has been modelled with Simscape. The simulation has been run at a fixed step size of $10^{-8}$ with the ode4 (Runge-Kutta) solver. The power converter model includes non ideal effects such as conduction and switching losses in the power switches, resistive losses in the transformer windings, and reactive components. Moreover, the branches of the full-bridge converters include the required dead time $(1 \mu \mathrm{s})$ to avoid bus voltage short circuits, which can not be avoided in real implementations. The system parameters are collected in Table I.
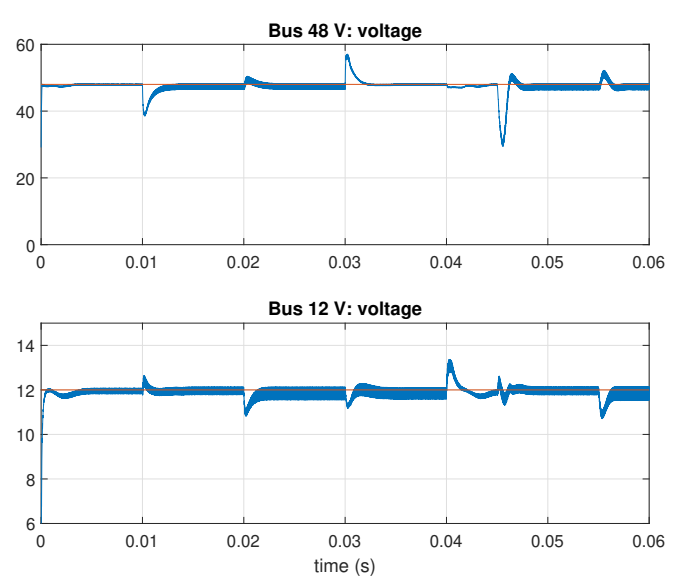

Fig. 6. Bus voltage (blue) and voltage reference (red). Top: $48 \mathrm{~V}$ bus; bottom: $12 \mathrm{~V}$ bus.

In order to check the system performance, several resistive load transients have been produced. Specifically,

$$
\begin{aligned}
& R_{2}= \begin{cases}5 \Omega & t \in[0,0.01) \cup[0.03,0.06) s \\
1 \Omega & t \in[0.01,0.03) s\end{cases} \\
& R_{3}= \begin{cases}3 \Omega & t \in[0,0.02) \cup[0.04,0.06) s \\
10 \Omega & t \in[0.02,0.04) s\end{cases}
\end{aligned}
$$

In turn, CPLs have also been connected to the buses through first order transfer functions with time constant $0.0001 \mathrm{~s}$, the transients being:

$$
\begin{aligned}
& P_{2}= \begin{cases}0 & t \in[0,0.045) s \\
2 k W & t \in[0.045,0.06) s\end{cases} \\
& P_{3}= \begin{cases}0 & t \in[0,0.055) s \\
100 W & t \in[0.055,0.06) s\end{cases}
\end{aligned}
$$

Figure 6 depicts the bus voltages tracking their respective references, showing the stability and robustness of the control design. Performance is also observed there and in Figure 7, which plots the relative voltage errors in each bus.

The control action variables, pictured in the top part of Figure 8 , are shown to react in a bounded way to counteract the effect of the sudden load and CPL changes in the bus voltages, while the output currents, $-i_{p 2},-i_{p 3}$ (recall Figure 2 ), are portrayed in the bottom part. In turn, the flux at the core of the transformer depicted in Figure 9 shows a zero mean value, which indicates the absence of saturation issues.

Finally, it is worth mentioning that changing the inductance factor of the primary winding in the converter model to

TABLE I

PARAMETERS FOR SYSTEM (2)

\begin{tabular}{||c|c||c|c||}
\hline$E_{1}$ & $400 \mathrm{~V}$ & $\omega_{1}$ & $2 \pi 40.0 e 3 \mathrm{rad} / \mathrm{s}$ \\
\hline$C_{2}$ & $200 \mu \mathrm{F}$ & $C_{3}$ & $600 \mu \mathrm{F}$ \\
\hline$\alpha_{12}$ & 0.12 & $\alpha_{13}$ & 0.03 \\
\hline$\alpha_{23}$ & 0.25 & $\alpha_{32}$ & $(0.25)^{-1}$ \\
\hline$L_{12}^{\prime}$ & $16.8 \mu \mathrm{H}$ & $L_{13}^{\prime}$ & $196 \mu \mathrm{H}$ \\
\hline$L_{23}^{\prime}$ & $4 \mu \mathrm{H}$ & $L_{32}^{\prime}$ & $4 \cdot(0.25)^{-2} \mu \mathrm{H}$ \\
\hline
\end{tabular}



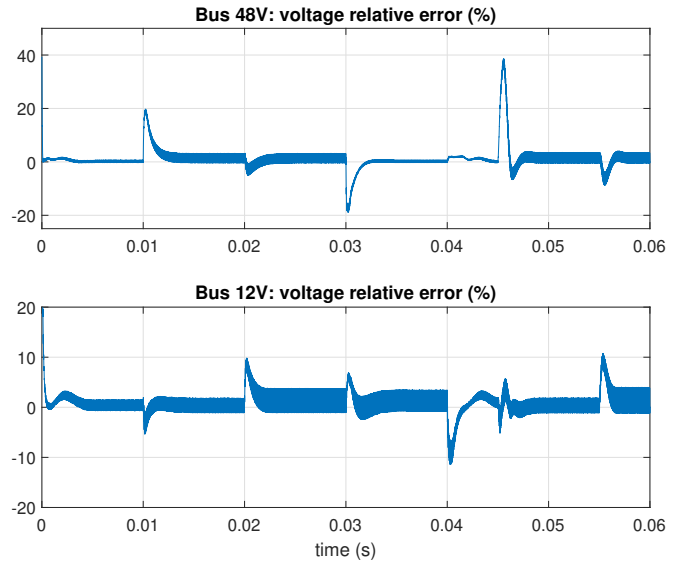

Fig. 7. Bus voltage relative error (\%). Top: $48 \mathrm{~V}$ bus; bottom: $12 \mathrm{~V}$ bus.

$L_{12}^{\prime}=21.8 \mu \mathrm{H}$, instead of the nominal $16.8 \mu \mathrm{H}$ indicated in Table I, without updating the control parameter, yields essentially the same outcome. This results confirms a good level of controller robustness. For the sake of brevity, and aiming at illustrating this behavior as well, the above presented simulation results are those obtained with $L_{12}^{\prime}=21.8 \mu \mathrm{H}$.

\section{Vi. Conclusions}

A feedback linearization-based controller was designed for a magnetically coupled dc-dc converter. The system was linearized and decoupled using an appropriate change of control and state variables, which allowed to design a simple, yet efficient, conventional PI controller. The inversion of the nonlinear control transformation was carried out using linear approximations, and no significant performance loses were observed. Realistic numerical simulations confirmed the stability and robustness of the system in the face of resistive load changes and presence of CPLs.

Further research is currently oriented to the physical implementation of the proposed controller in a laboratory prototype.
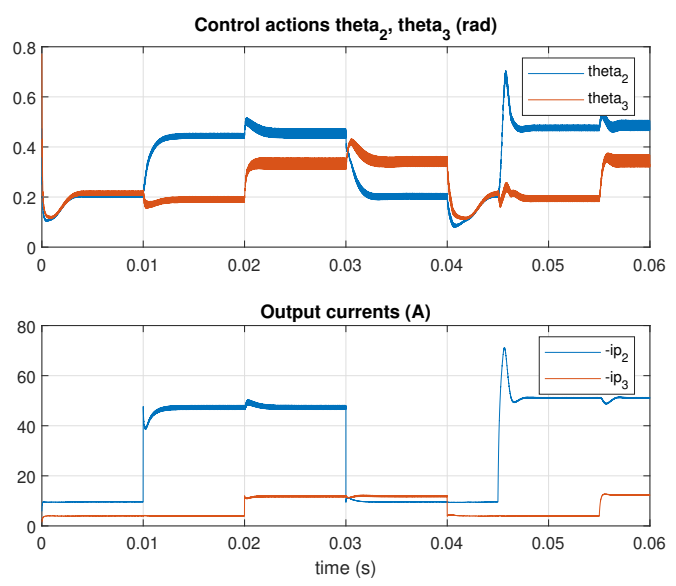

Fig. 8. Top: control actions $\theta_{2}$ (blue), $\theta_{3}$ (red) in radiants; bottom: output currents $-i_{p 2}$ (blue), $-i_{p 3}$ (red) in Ampères.
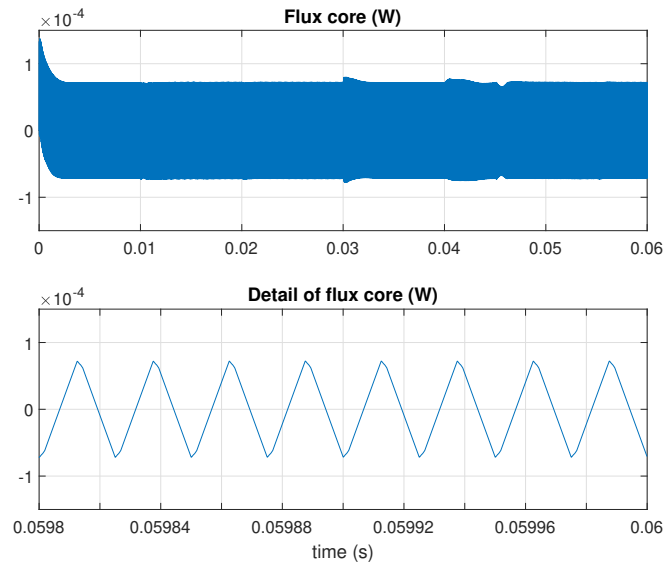

Fig. 9. Top: flux at the core of the transformer; bottom: detail.

\section{ACKNOWLEDGMENT}

The work was partially supported by the Government of Spain through the Agencia Estatal de Investigación Project DPI2017-85404-P and by the Generalitat de Catalunya through the Project 2017 SGR 872.

\section{REFERENCES}

[1] "Use of new energies in road transport," European Green Vehicles Initiative. PPP, Tech. Rep., 2013.

[2] "Hybrid and electric vehicles - the electric drive commutes," International Energy Agency, Tech. Rep., 2016.

[3] C. Chan, "The state of the art of electric, hybrid, and fuel cell vehicles," Proc. of the IEEE, vol. 95, no. 4, pp. 704-718, 2007.

[4] O. Coppin, "From 12+12V to 48V: a new road map for hybridization," in Proc. Engine Expo 2016, 2016.

[5] B. Farhangi and H. Toliyat, "Modeling and analyzing multiport isolation transformer capacitive components for onboard vehicular power conditioners," IEEE Trans. on Industrial Electronics, vol. 62, no. 5, pp. 3134-3142, 2015.

[6] B. Karanayil, M. Ciobotaru, and V. Agelidis, "Power flow management of isolated multiport converter for more electric aircraft," IEEE Trans. on Power Electronics, vol. 32, no. 7, pp. 5850-5861, 2017.

[7] Z. Qian, "Modeling and design of multi-port dc/dc converters," Ph.D. dissertation, University of Central Florida, 2010.

[8] H. Tao, J. Duarte, and M. Hendrix, "Three-port triple-half-bridge bidirectional converter with zero-voltage switching," IEEE Trans. on Power Electronics, vol. 23, no. 2, pp. 782-792, 2008.

[9] H. Matsuo, W. Lin, F. Kurokawa, T. Shigemizu, and N. Watanabe, "Characteristics of the multiple-input DC-DC converter," IEEE Trans. on Industrial Electronics, vol. 51, no. 3, pp. 625 - 631, 2004.

[10] V. Jakka, A. Shukla, and G. Demetriades, "Dual-transformer-based asymmetrical triple-port active bridge (DT-ATAB) isolated DC-DC converter," IEEE Trans. on Industrial Electronics, vol. 64, no. 6, pp. 4549-4560, 2017.

[11] Z. Li, Y. Wang, L. Shi, J. Huang, Y. Cui, and W. Lei, "Generalized averaging modeling and control strategy for three-phase dual-activebridge DC-DC converters with three control variables," in Proc. IEEE Applied Power Electronics Conference and Exposition, 2017.

[12] C. Zhao, S. Round, and J. Kolar, "An isolated three-port bidirectional DC-DC converter with decoupled power flow management," IEEE Trans. on Power Electronics, vol. 23, no. 5, pp. 2443-2453, 2008.

[13] K. Xiangli, S. Li, and K. Smedley, "Decoupled PWM plus phase-shift control for a dual-half-bridge bidirectional DC-DC converter," IEEE Trans. on Power Electronics, vol. 33, no. 8, pp. 7203-7213, 2018.

[14] B. Choi, W. Lim, S. Choi, and J. Sun, "Comparative performance evaluation of current-mode control schemes adapted to asymmetrically driven bridge-type pulsewidth modulated DC-to-DC converters," IEEE Trans. on Industrial Electronics, vol. 55, no. 5, pp. 2033-2042, 2008. 
[15] Z. Qian, O. Abdel-Rahman, H. Al-Atrash, and I. Batarseh, "Modeling and control of three-port DC/DC converter interface for satellite applications," IEEE Trans. on Power Electronics, vol. 25, no. 3, pp. 637-649, 2010.
[16] R. Griñó and A. Dòria-Cerezo, "Modelling and simulation of a magnetically coupled multiport dc-dc converter," in Proc. 24th Int. Conf. on Emerging Technologies and Factory Automation, 2019. 